\title{
Thermal emittance from ionization-induced trapping in plasma accelerators
}

\author{
C. B. Schroeder, ${ }^{1}$ J.-L. Vay, ${ }^{1}$ E. Esarey, ${ }^{1}$ S. S. Bulanov, ${ }^{2,1}$ C. Benedetti, ${ }^{1}$ L.-L. Yu, ${ }^{3}$ \\ M. Chen, ${ }^{3}$ C. G. R. Geddes, ${ }^{1}$ and W. P. Leemans ${ }^{1,2}$ \\ ${ }^{1}$ Lawrence Berkeley National Laboratory, Berkeley, California 94720, USA \\ ${ }^{2}$ Department of Physics, University of California, Berkeley, California 94720, USA \\ ${ }^{3}$ Key Laboratory for Laser Plasmas (Ministry of Education), Department of Physics and Astronomy, \\ Shanghai Jiao Tong University, Shanghai 200240, China
}

(Received 19 June 2014; published 3 October 2014)

\begin{abstract}
The minimum obtainable transverse emittance (thermal emittance) of electron beams generated and trapped in plasma-based accelerators using laser ionization injection is examined. The initial transverse phase space distribution following ionization and passage through the laser is derived, and expressions for the normalized transverse beam emittance, both along and orthogonal to the laser polarization, are presented. Results are compared to particle-in-cell simulations. Ultralow emittance beams can be generated using laser ionization injection into plasma accelerators, and examples are presented showing normalized emittances on the order of tens of $\mathrm{nm}$.
\end{abstract}

DOI: 10.1103/PhysRevSTAB.17.101301

PACS numbers: 52.38.Kd, 32.80.Fb

\section{INTRODUCTION}

Plasma-based accelerators [1] can produce extremely large accelerating gradients, enabling compact sources of high energy particle beams. The characteristic accelerating field in a plasma accelerator is on the order of $E_{0}(\mathrm{~V} / \mathrm{m}) \simeq 96 \sqrt{n_{0}\left(\mathrm{~cm}^{-3}\right)}$, with $n_{0}$ the plasma electron density, and $E_{0}$ can be several orders of magnitude larger than that obtainable in conventional accelerators. In addition, plasma accelerators produce ultrashort electron beams with durations $<\lambda_{p} / c$ [2], where $\lambda_{p}(\mu \mathrm{m}) \simeq 3.3 \times 10^{10} /$ $\sqrt{n_{0}\left(\mathrm{~cm}^{-3}\right)}$ is the plasma wavelength. High-quality $\mathrm{GeV}$ electron beams were first demonstrated using an intense laser driving a plasma wave in a $\mathrm{cm}$-scale plasma, relying on self-injection for electron beam generation [3]. In the nonlinear laser-driven bubble regime [4], where the particles are self-trapped [5], experiments show that an electron beam with normalized transverse emittance of $\epsilon_{n} \sim 0.1 \mathrm{~mm} \mathrm{mrad}$ can be achieved [6]. High-quality, laser-plasma-accelerated electron beams could be good candidates to drive free-electron lasers $[7,8]$ or Thomson photon sources [9], enabling a new generation of low-cost, compact light sources. Improved beam phase space characteristics, and, in particular, reduced transverse emittance, is highly desired for light sources and other applications.

In order to improve the quality and stability of laserplasma-accelerated electron beams, controlled injection methods are actively being pursued, including colliding pulse injection $[10,11]$, plasma density transitions $[12,13]$,

Published by the American Physical Society under the terms of the Creative Commons Attribution 3.0 License. Further distribution of this work must maintain attribution to the author(s) and the published article's title, journal citation, and DOI. and ionization injection [14-23]. In conventional ionization injection into laser-plasma accelerators [16-18], a single laser pulse is used both for wake generation and ionization of a high-Z gas. Typically large laser intensities $a_{0}>1$, with $w_{0} \sim 10 \mu \mathrm{m}$, are needed to excite a sufficiently large wake so that an electron ionized near the peak intensity of the laser pulse can be trapped. Here $w_{0}$ is the laser spot size and $a_{0}$ is the peak normalized amplitude of the laser vector potential. For linear polarization, $a_{0}^{2}=7.3 \times$ $10^{-19}[\lambda(\mu \mathrm{m})]^{2} I_{0}\left(\mathrm{~W} / \mathrm{cm}^{2}\right)$ with $I_{0}$ the peak laser intensity and $\lambda$ the laser wavelength. Ionization at these large laser intensities typically results in large transverse momentum [18], and, hence, large transverse emittance of the injected particle beam.

The trapped electron beam quality may be improved by considering independently controlled ionization injection into a preestablished plasma wake. For example, one may consider a charged particle beam driving a plasma wake in the blow-out regime [24], followed by a laser pulse that ionizes and traps electrons in the wake of the beam [20,21]. Since field ionization by the beam driver can be small, this reduces the intensity required for the ionization laser, and simulations of this method indicate that ultralow emittance beams can be generated [20,21]. An all-optical method of reducing the ionization laser intensity has also been proposed that relies on using two lasers of different colors [22]. In the two-color ionization injection method, a longwavelength pump pulse, with large ponderomotive force and small peak electric field, excites a wake without fully ionizing a high- $Z$ gas. A short-wavelength injection pulse, with small ponderomotive force and large peak electric field, copropagating and delayed with respect to the pump laser, ionizes a fraction of the remaining bound electrons at a trapping phase of the plasma wave, generating an electron beam that is accelerated by the wake. Numerical modeling 


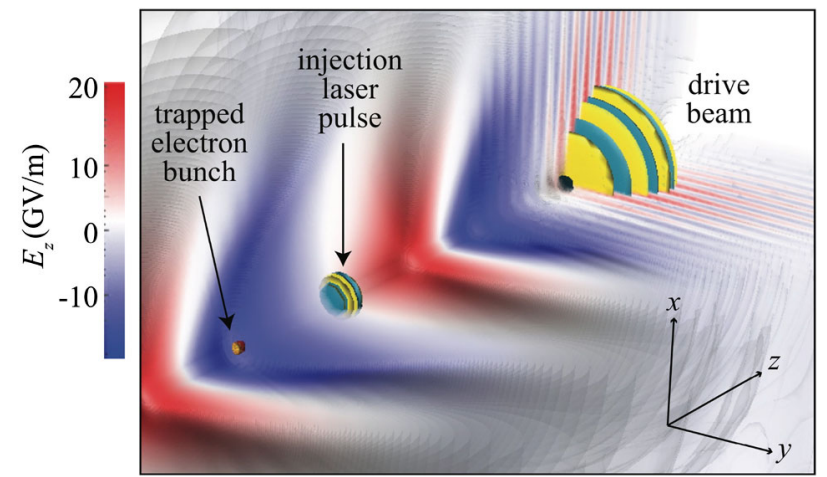

FIG. 1. Illustration of (two-color) laser-ionization injection from a 3D PIC simulation: a drive beam (a circular-polarized, $5 \mu \mathrm{m}$-wavelength laser pulse with $a_{0}=1.17,92$ fs FWHM duration, and spot size $w_{0}=36 \mu \mathrm{m}$ ) is propagating in the $\hat{z}$ direction in a plasma of density $n_{0}=2 \times 10^{17} \mathrm{~cm}^{-3}$ doped with a high- $Z$ gas (Krypton), and generates a plasma wave (wake), $E_{z}$. An injection laser pulse (linear-polarized with $\lambda_{i}=0.4 \mu \mathrm{m}$, $a_{i}=0.135,16$ fs FWHM duration, and spot size $w_{i}=5 \mu \mathrm{m}$ ) is copropagating and delayed with respect to the beam driving the plasma wave such that ionized electrons $\left(\mathrm{Kr}^{8+}\right.$ to $\left.\mathrm{Kr}^{9+}\right)$ are trapped in the wake forming a trailing accelerated electron bunch.

indicates that two-color ionization injection can produce electron beams with normalized emittances of the order $\sim 10 \mathrm{~nm}$ [22]. An illustration of the basic geometry of the two-color ionization injection method is shown in Fig. 1.

In this work we address the fundamental limit of the obtainable beam emittance, the thermal emittance, from laser ionization injection methods in plasma-based accelerators. These results are independent of the driver of the plasma accelerator (e.g., laser or particle beam). In particular, it is shown that the thermal emittance of the beam at injection, in the plane of laser polarization, is approximately $\epsilon_{x} \simeq(3 \pi / \sqrt{2})\left(r_{\mathrm{e}} / \alpha^{4}\right)\left(U_{I} / U_{H}\right)^{-3 / 2} w_{i} a_{i}^{2} / \lambda_{i}$, where $r_{\mathrm{e}}$ is the classical electron radius, $\alpha$ is the fine structure constant, $U_{I}$ is the ionization potential (normalized to $U_{H} \simeq 13.6 \mathrm{eV}$ ), and $a_{i}^{2}, w_{i}$, and $\lambda_{i}$ are the normalized intensity, spot size, and wavelength of the ionization injection laser pulse, respectively.

This paper is organized as follows. In Sec. II we describe and calculate the spatial distribution of electrons created by laser tunneling ionization. The initial distribution in longitudinal position determines the initial transverse momentum of the electrons obtained from the laser quiver motion in the plane of laser polarization. The initial phase space distribution of the ionized electron beam is compared to particle-in-cell (PIC) simulations. Section III describes the effect of the ponderomotive force of the ionization laser. It is shown that, since the momentum gain via the ponderomotive force is correlated to the transverse position of the electron at ionization, the ponderomotive force does not contribute to the initial thermal emittance of the beam. However, the laser ponderomotive force can contribute to the saturated emittance after phase mixing. In Sec. IV, the thermal emittance from ionization injection is derived. Interaction with the plasma wave will typically lead to phase mixing and emittance growth. Expressions for the transverse emittance, after phase mixing, along and orthogonal to the laser polarization direction are derived. These results are compared to 3D PIC simulations of the ionization injected electron beam using two-color ionization injection. Conclusions are presented in Sec. V.

\section{ELECTRON PHASE SPACE DISTRIBUTION FROM LASER TUNNELING IONIZATION}

The laser ionization process determines the initial electron phase space distribution. For a linearly polarized laser field, the ionization rate in the tunneling limit, assuming the static electric field approximation, is [25]

$$
W=4 \omega_{a} C \frac{\left(U_{I} / U_{H}\right)^{\left(6 n^{*}-3|m|-1\right) / 2}}{\left|E / E_{a}\right|^{2 n^{*}-|m|-1}} \exp \left[-\frac{2\left(U_{I} / U_{H}\right)^{3 / 2}}{3\left|E / E_{a}\right|}\right]
$$

where $\omega_{a}=\alpha^{3} c / r_{e}, U_{I}$ is the ionization potential with $U_{H}=m_{e} c^{2} \alpha^{2} / 2=13.6 \mathrm{eV}$ the ionization potential of hydrogen, $E$ is the laser field, and $E_{a}=e / r_{B}^{2} \simeq$ $0.5 \mathrm{TV} / \mathrm{m}$ the atomic field, with $r_{B}=r_{e} / \alpha^{2}$ the Bohr radius, $r_{e}=e^{2} / m_{e} c^{2}$ the classical electron radius, and $\alpha=e^{2} / \hbar c$ the fine structure constant. Here $m$ is the projection of the angular momentum quantum number $l$ on the electric wave field and $n^{*}=Z\left(U_{H} / U_{I}\right)^{1 / 2}$ is the effective principal quantum number, where $Z$ is the ion charge number after ionization. The constant $C$ is a function of the atomic quantum numbers (determined by the atomic wave function),

$$
C=\frac{2^{4 n^{*}-4-2|m|}}{n^{*} \Gamma\left(n^{*}+l^{*}+1\right) \Gamma\left(n^{*}-l^{*}\right)} \frac{(2 l+1)(l+|m|) !}{|m| !(l-|m|) !},
$$

where $l^{*}=n_{0}^{*}-1$ is the effective orbital quantum number, with $n_{0}^{*}$ the effective principal quantum number of the ground state. For a hydrogen-like atom, $C=1$. Equation (1) will be valid for small Keldysh parameter, $\gamma_{\mathrm{K}}=\left(U_{I} / 2 U_{p}\right)^{1 / 2}<1$, where $U_{p}=m_{e} c^{2} a_{i}^{2} / 4$ is the electron oscillation energy, with $a_{i}=e A_{i} / m_{e} c^{2}$ the peak normalized laser vector potential of the linear-polarized ionization laser. For $\gamma_{\mathrm{K}}>1$ multiphoton ionization will be dominant. Ionization injection into a plasma wakefield can be achieved using multiphoton ionization. However, for typical laser-plasma parameters $\gamma_{\mathrm{K}}<1$, and in the following we consider laser tunneling ionization to be the dominant ionization mechanism with $\gamma_{\mathrm{K}}<1$.

The distribution of ionized electrons can be calculated from the laser field using the ionization rate Eq. (1). Consider the laser field used for ionization injection to have the form 


$$
E_{i}=E_{p} \exp \left(-r^{2} / w_{i}^{2}\right) \cos \left(k_{i} \xi\right)
$$

where $\xi=z-c t$ is the comoving variable, $w_{i}$ is the laser spot size, $k_{i}=2 \pi / \lambda_{i}$, with $\lambda_{i}$ the laser wavelength, and $E_{p}$ is the peak electric field of the laser $E_{p}[\mathrm{TV} / \mathrm{m}] \simeq$ $3.21 a_{i} / \lambda_{i}[\mu \mathrm{m}]$. From Eq. (1), the ionization probability is largest near the peaks of the laser field where $r \ll w_{i}$ and $\left|k_{i} \xi-j \pi\right| \ll 1$, with $j$ an integer. Here we assume that ionization occurs near the peak of the laser envelope (laser envelope effects will be discussed in Sec. II B). Assuming the charge state of the gas/ions is not fully ionized (a fully ionized gas will be discussed in Sec. II D), near the peak of the laser electric field,

$$
E_{L} \simeq E_{p}\left[1-\left(r / w_{i}\right)^{2}-\left(k_{i} \xi-j \pi\right)^{2} / 2\right],
$$

and, using Eq. (1), the ionization rate is

$$
W \approx W_{p} \exp \left[-\frac{\left(r / w_{i}\right)^{2}}{\sigma_{\psi}^{2}}\right] \exp \left[-\frac{\left(k_{i} \xi-j \pi\right)^{2}}{2 \sigma_{\psi}^{2}}\right]
$$

In Eq. (5), $W_{p}$ is approximately the tunneling ionization rate at the peak of the laser field and the rms spread in injection laser phase is

$$
\sigma_{\psi}=\Delta\left[1-\left(2 n^{*}-|m|-1\right) \Delta^{2}\right]^{-1 / 2},
$$

with

$$
\Delta=\left(\frac{3}{2} \frac{E_{p}}{E_{a}}\right)^{1 / 2}\left(\frac{U_{H}}{U_{I}}\right)^{3 / 4}=\left(\frac{3 \pi r_{e} a_{i}}{\alpha^{4} \lambda_{i}}\right)^{1 / 2}\left(\frac{U_{H}}{U_{I}}\right)^{3 / 4} .
$$

The rms spread in laser phase position of the ionized electrons about the field peak, $\sigma_{\psi}$, will result in a finite transverse momentum spread of the electrons after exiting the laser, as discussed in Sec. II A.

The parameter $\Delta^{2}$ is the normalized laser field amplitude. Ionization injection is optimized for field amplitudes near the threshold for ionization. For short-pulse lasers, the field amplitude required to ionize an atomic state can be estimated by considering the laser field amplitude where the ionization rate (probability) over a laser period is of order unity: $\left(\lambda_{i} / c\right) W_{p} \sim 1$. For typical parameters of interest $\left(\lambda_{i} / c\right) W_{p} \sim 1$ for $\Delta^{2} \ll 1$. For example, $\left(\lambda_{i} / c\right) W_{p}=1$ requires $\Delta^{2} \simeq 0.07$ for $\mathrm{He}^{+}\left(U_{I}=24.6 \mathrm{eV}\right)$ with $\lambda_{i}=0.8 \mu \mathrm{m},\left(\lambda_{i} / c\right) W_{p}=1$ requires $\Delta^{2} \simeq 0.1$ for $\mathrm{N}^{6+}\left(U_{I}=522 \mathrm{eV}\right)$ with $\lambda_{i}=0.8 \mu \mathrm{m}$, and $\left(\lambda_{i} / c\right) W_{p}=1$ requires $\Delta^{2} \simeq 0.04$ for $\mathrm{Kr}^{9+}\left(U_{I}=230 \mathrm{eV}\right)$ with $\lambda_{i}=$ $0.4 \mu \mathrm{m}$. Hence, the limit $\Delta^{2} \ll 1$ is satisfied at the ionization threshold and $\sigma_{\psi} \simeq \Delta<1$ (i.e., ionization occurs near the field maxima).

\section{A. Intrinsic transverse momentum spread}

We make the semiclassical assumption that the initial transverse momentum of the electrons is zero, with respect to the atom, at the moment of their ionization. Assuming an underdense plasma and $k_{i} w_{i} \gg 1$, transverse canonical momentum conservation [1] implies $u_{x}-a_{x} \simeq-a_{x}\left(t_{i}\right)$, where $u_{x}$ is the electron momentum normalized to $m_{e} c$, $t_{i}$ is the ionization time, and the $x$ direction is in the plane of laser polarization. After exiting the ionization laser, the transverse momentum of the electron is given by the laser vector potential at ionization

$$
u_{x}=-a_{x}\left(t_{i}\right) \simeq-a_{i} \sin \left[k_{i} \xi\left(t_{i}\right)\right] e^{-r\left(t_{i}\right)^{2} / w_{i}^{2}},
$$

where $\left|k_{i} \xi\left(t_{i}\right)-j \pi\right| \ll 1$ is near the peak of the laser electric field at the time of ionization $t_{i}$. Ionization away from the peak of the laser electric field results in transverse momentum along the direction of laser polarization after the electron exits the laser. The rms spread in transverse momentum $\sigma_{p_{x}}=\left\langle u_{x}^{2}\right\rangle^{1 / 2}=a_{i}\left\langle\sin ^{2}\left(k_{i} \xi\right) \exp \left(-2 r^{2} / w_{i}^{2}\right)\right\rangle^{1 / 2}$ (in the laser polarization plane) is

$$
\sigma_{p_{x}}=a_{i}\left(\frac{e^{-\sigma_{\psi}^{2}} \sinh \left(\sigma_{\psi}^{2}\right)}{1+2 \sigma_{\psi}^{2}}\right)^{1 / 2} \simeq \sigma_{\psi}\left(1-3 \sigma_{\psi}^{2} / 2\right) a_{i}
$$

where $\sigma_{\psi}$ is the rms spread in laser phase position of the ionized electrons about the field peak Eq. (6). Equation (9) includes the effects of the nonlinear laser phase dependence on momentum and off-axis ionization of electrons. For $\sigma_{\psi} \ll 1$,

$$
\sigma_{p_{x}} \simeq a_{i} \Delta \simeq\left(\frac{3 \pi r_{e}}{\alpha^{4} \lambda_{i}}\right)^{1 / 2}\left(\frac{U_{H}}{U_{I}}\right)^{3 / 4} a_{i}^{3 / 2} .
$$

The rms momentum spread, in the laser polarization plane, has a nonlinear dependence on the ionization laser intensity $\sigma_{p_{x}} \propto a_{i}^{3 / 2}$. Note that the derivation of the differential ionization rate with respect to the initial photoelectron momentum (see Ref. [25]) yields the rms momentum spread along the direction of the field (to lowest order in $\gamma_{\mathrm{K}} \ll 1$ for a plane electromagnetic wave) of $\left[3 \hbar \omega /\left(2 m_{e} c^{2}\right)\right]^{1 / 2} \gamma_{\mathrm{K}}^{-3 / 2}=$ $a_{i} \Delta$, in agreement with Eq. (10).

Equation (8) assumes the electrons are ionized from atoms at rest. The ion temperature may be neglected provided the ion velocity $\beta_{i}=v_{i} / c$ is small compared to $\sigma_{p x}$, where $\beta_{i} \simeq\left(2 k_{\mathrm{B}} T_{i} / M_{i} c^{2}\right)^{1 / 2}$, with $M_{i}$ the ion mass, $T_{i}$ the ion temperature, and $k_{\mathrm{B}}$ the Boltzmann constant. The condition $\beta_{i} \ll \sigma_{p x}$ is typically well satisfied for parameters of interest.

\section{B. Laser envelope effects}

Since the rate of laser ionization grows exponentially about the laser electric field peak [cf. Eq. (1)], laser envelope effects manifest as small corrections to the 
distribution of ionized electrons. Consider a Gaussian laser envelope along the propagation direction such that

$$
E_{i}=E_{p} e^{-\xi^{2} / 2 L_{i}^{2}} \cos \left(k_{i} \xi\right),
$$

where $L_{i}$ is the rms length of the laser field envelope. Assuming the ionization occurs near the peak of the laser envelope $\xi^{2} \ll L_{i}^{2}$ (and the peaks of the laser electric field), such that $E_{i} \simeq E_{p}\left(1-\xi^{2} / 2 L_{i}^{2}\right)$, the rate of ionization at the field peak located at $\xi$ in the laser envelope is

$$
W=W_{p} \exp \left[-\xi^{2} /\left(2 \Delta^{2} L_{i}^{2}\right)\right]
$$

The rate of ionization away from the laser envelope peak decreases, and the distribution has rms length $L_{i} \Delta$. Figure 2 shows the normalized ionization rate (solid black line), the laser envelope (dotted blue line), and Eq. (12) describing the reduced rate of ionization owing to the laser envelope (dashed red line), for a Gaussian envelope with the parameters $k_{i} L_{i}=64$ and $\Delta=0.2$. As illustrated in Fig. 2 the ionization rate decreases exponentially over a fraction of the laser envelope.

Ionization that occurs at a field peak away from the peak of the laser envelope will be at a smaller laser field $a(\xi)$, resulting in a correspondingly smaller injection laser phase region $\sigma_{\psi} \propto[a(\xi)]^{1 / 2}$. Assuming $k_{i} L_{i} \Delta \gg 1$, and performing an average over the field peaks weighted by the local ionization rate, the rms spread in the laser phase region of the ionized electrons is

$$
\sigma_{\psi}=\Delta\left[1+\left(2 n^{*}-|m|-1\right) \Delta^{2} / 2-\Delta^{2} / 4\right],
$$

where the last term of the right-hand side of Eq. (13) is due to laser envelope effects (reducing the injection phase

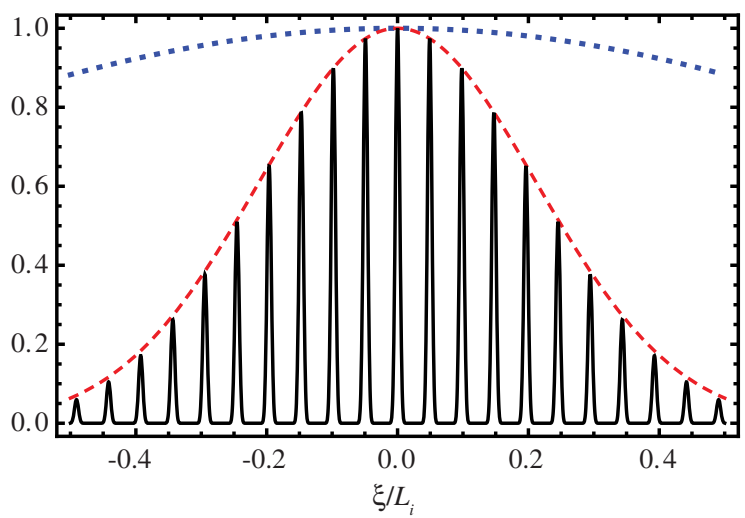

FIG. 2. Solid black curve is the normalized ionization rate for a laser field having a Gaussian envelope with the parameters $k_{i} L_{i}=64$ and $\Delta=0.2$. The rms width of each peak is $\sigma_{\xi}=\sigma_{\psi} / k_{i} \simeq \Delta / k_{i}$. Dashed red curve shows the longitudinal region of ionization due to the laser envelope, with rms width $\sigma_{\xi}=L_{i} \Delta$. Dotted blue line is the normalized laser field envelope with rms length $\sigma_{\xi}=L_{i}$. region). Notice that the correction due to the laser envelope is independent of the laser envelope length $L_{i}$, since the rms length of the ionization region is proportional to the envelope length $L_{i} \Delta$, and Eq. (13) is valid for any Gaussian envelope length, provided $k_{i} L_{i} \Delta \gg 1$.

Including envelope effects the rms spread in transverse momentum after exiting the laser is $\sigma_{p_{x}}=$ $\left\langle\left\langle a \sigma_{\psi}\left(1-3 \sigma_{\psi}^{2} / 2\right)\right\rangle\right\rangle$, where the average, denoted by $\langle\langle\cdot\rangle\rangle$, is over the field peaks weighted by the local ionization rate. The Gaussian laser envelope reduces the rms transverse momentum of the ionized electrons because laser phase fronts away from the peak have lower field amplitude and, hence, the electrons are ionized over a narrower range of laser phases. Performing the weighting yields,

$$
\sigma_{p_{x}}=a_{i} \Delta\left\{1-\frac{3}{4} \Delta^{2}-\frac{3}{2} \Delta^{2}+\left[n^{*}-(|m|+1) / 2\right] \Delta^{2}\right\}
$$

where the second, third, and fourth terms in the brackets are the effects of the laser envelope, the nonlinear dependence of momentum on phase position and off-axis ionization [cf. Eq. (9)], and the nonexponential dependence on the ionization rate [cf. Eq. (6)], respectively.

Figure 3 shows the rms transverse momentum distribution versus ionization laser intensity for two cases: (a) conventional single-pulse ionization injection using a high intensity laser pulse (cf. parameters used in Ref. [18]), and (b) two-color ionization injection using a low intensity laser pulse (cf. parameters used in Ref. [22]). Figure 3(a) is for an ionization laser with $\lambda_{i}=$ $0.8 \mu \mathrm{m}$ and FWHM duration $40 \mathrm{fs}$, ionizing a nitrogen gas, $\mathrm{N}^{5+} \rightarrow \mathrm{N}^{6+}\left(U_{I}=552 \mathrm{eV}\right)$, in a background plasma of density $1.7 \times 10^{18} \mathrm{~cm}^{-3}$ (for this case $\Delta \approx 0.3$ ), and Fig. 3(b) is for an ionization laser with $\lambda_{i}=0.4 \mu \mathrm{m}$ and FWHM duration 16 fs, ionizing a krypton gas, $\mathrm{Kr}^{8+} \rightarrow$ $\mathrm{Kr}^{9+}\left(U_{I}=230 \mathrm{eV}\right)$, in a background plasma of density $2 \times 10^{17} \mathrm{~cm}^{-3}$ (for this case $\Delta \approx 0.2$ ). The points in Fig. 3 were obtained from two-dimensional (2D) PIC simulations with the laser polarization out of the simulation plane: Figure 3(a) used the PIC code VLPL [26] (implementing the ionization algorithm Ref. [27]), and the Fig. 3(b) used the PIC code WARP [28] (implementing the ionization algorithm Ref. [27]). For large laser intensities $a \sim 1$ [as in Fig. 3(a)], the 2D PIC simulation with the laser polarization out of the plane allows separation of the effects of the quiver motion and the ponderomotive force (the effect of the ionization laser ponderomotive force is discussed in Sec. III). The dashed curves show Eq. (14) for each case. Excellent agreement between the PIC modeling and the analytic expression Eq. (14) is achieved for a range of laser intensities, different laser wavelengths, and for different gases. 

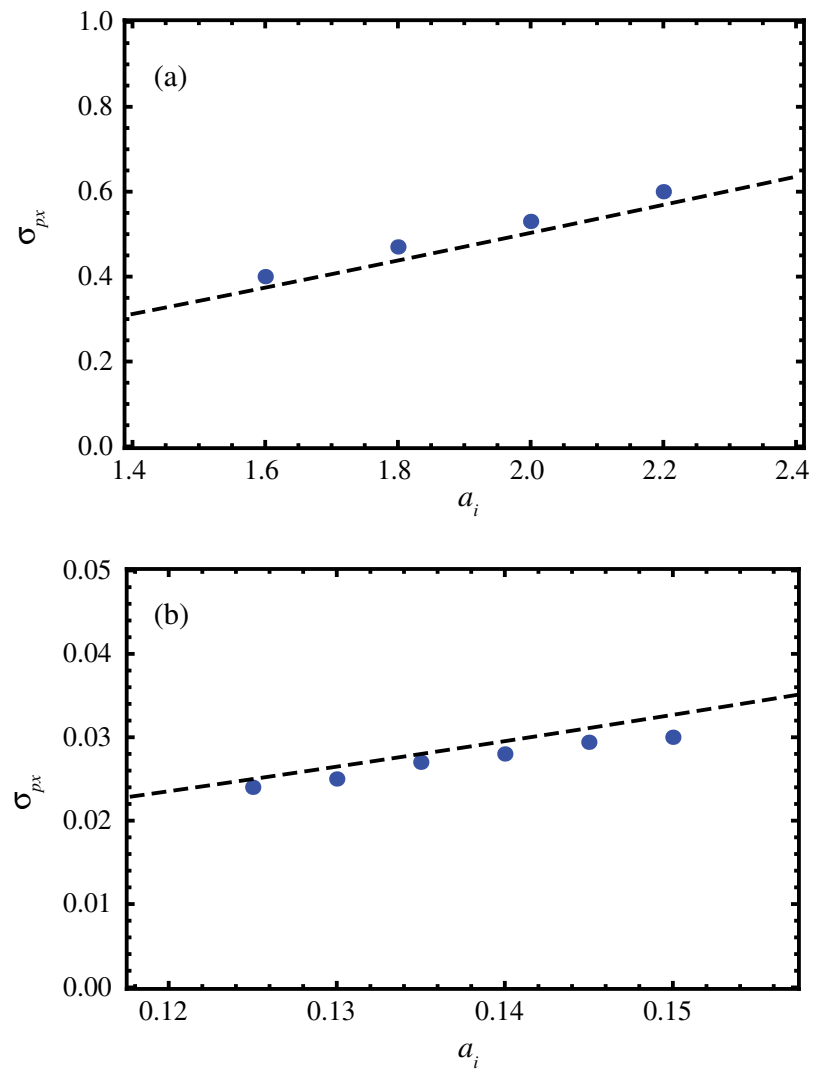

FIG. 3. The rms transverse momentum distribution versus ionization laser intensity for laser ionization injection using (a) a high intensity laser pulse, with $\lambda_{i}=0.8 \mu \mathrm{m}$ and FWHM duration $40 \mathrm{fs}$, ionizing a nitrogen gas, $\mathrm{N}^{5+} \rightarrow \mathrm{N}^{6+}\left(U_{I}=552 \mathrm{eV}\right)$, in a background plasma of density $1.7 \times 10^{18} \mathrm{~cm}^{-3}$, and (b) a low intensity laser pulse, with $\lambda_{i}=0.4 \mu \mathrm{m}$ and FWHM duration $16 \mathrm{fs}$, ionizing a krypton gas, $\mathrm{Kr}^{8+} \rightarrow \mathrm{Kr}^{9+}\left(U_{I}=230 \mathrm{eV}\right)$, in a background plasma of density $2 \times 10^{17} \mathrm{~cm}^{-3}$. The points are obtained from PIC simulations, and the dashed curves are Eq. (14).

\section{Transverse electron distribution after ionization}

Equation (5) predicts that the rms spread in transverse position of ionized electrons is

$$
\sigma_{x}=\sigma_{y}=w_{i} \sigma_{\psi} / \sqrt{2}
$$

where $\sigma_{\psi}$, including laser envelope effects, is given by Eq. (13). In the limit $\Delta^{2} \ll 1, \sigma_{x}=\sigma_{y} \simeq w_{i} \Delta / \sqrt{2}$. As expected, the transverse size of the distribution of ionized electrons is proportional to the laser spot size, $\sigma_{y} \propto w_{i} a_{i}^{1 / 2}$. Figure 4 shows an example of the rms transverse size of the distribution of electrons ionized versus the laser spot size. The points in Fig. 4 are simulations performed using the PIC code WARP, and the dashed line is Eq. (15). For this example we are considering the two-color ionization injection geometry [22] with an ionization laser $a_{i}=0.135$, and all other parameters as in Fig. 3(b). For these parameters $\Delta \simeq 0.2$. There is excellent agreement between the

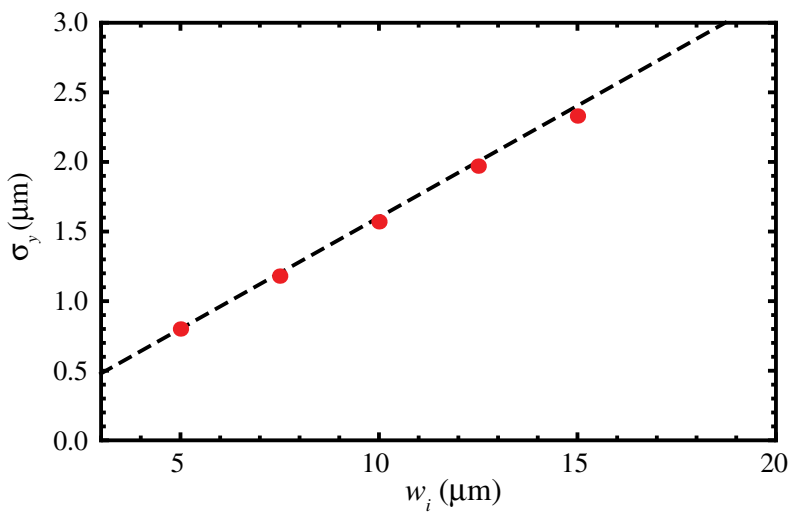

FIG. 4. The rms width of the transverse distribution of ionized electrons versus laser spot size $w_{i}$ using a laser with $a_{i}=0.135$ and $\lambda_{i}=0.4 \mu \mathrm{m}$ ionizing a krypton gas, $\mathrm{Kr}^{8+} \rightarrow \mathrm{Kr}^{9+}$ $\left(U_{I}=230 \mathrm{eV}\right)$. The points are PIC simulations using WARP, and the dashed line is the analytic expression Eq. (15).

expression Eq. (15) and the numerical modeling of the ionization.

\section{Fully ionized gas}

As the ionization laser propagates it generates plasma electrons at the rate

$$
\partial_{t}\left(n / n_{g}\right)=\left[1-\left(n / n_{g}\right)\right] W,
$$

where $n_{g}$ is the atomic gas density (of the ions in the charge state used for ionization trapping). The initial distribution of electrons will be given by the ionization rate distribution Eq. (5), provided that the ion charge state is not fully ionized before reaching the peak field of the laser envelope. Figure 5 shows the solution to Eq. (16) for the fractional ionization $n / n_{g}$ for (a) $a_{i}=0.14$, where the peak field of the laser is below that required to fully ionize on axis, and (b) $a_{i}=0.2$, where the peak field of the laser is sufficiently high to fully ionize before reaching the laser envelope peak. Here we are considering ionization of krypton gas, $\mathrm{Kr}^{8+} \rightarrow \mathrm{Kr}^{9+}\left(U_{I}=230 \mathrm{eV}\right)$, using a $0.4 \mu \mathrm{m}$ laser with length $k_{i} L_{i}=32$. As Fig. 5 illustrates, the distribution of ionized electrons is altered from a Gaussian form if the charge state is fully ionized before reaching the peak of the laser.

If the atomic state is fully ionized $\left(n / n_{g} \simeq 1\right)$ after the laser envelope reaches some threshold field before the laser envelope peak $a_{f}<a_{i}$, then we expect the transverse momentum of the ionized electrons to saturate at, using Eq. (10),

$$
\sigma_{p_{x}} \simeq\left(\frac{3 \pi r_{e}}{\alpha^{4} \lambda_{i}}\right)^{1 / 2}\left(\frac{U_{H}}{U_{I}}\right)^{3 / 4} a_{f}^{3 / 2}
$$

for $a_{f}<a_{i}$. For the parameters of Fig. 5 [and Fig. 3(b)], the gas is fully ionized for the state $\mathrm{Kr}^{8+} \rightarrow \mathrm{Kr}^{9+}$ at $a_{f} \simeq 0.15$, 

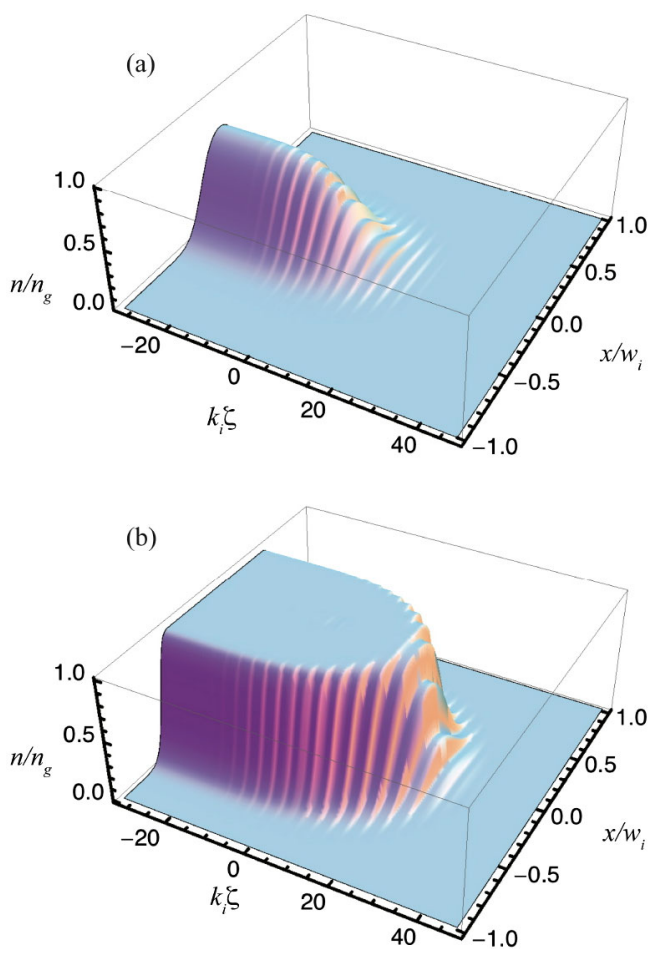

FIG. 5. Fractional ionization $n / n_{g}$ for (a) $a_{i}=0.14$ and (b) $a_{i}=0.2$. The laser field has a wavelength of $0.4 \mu \mathrm{m}$ and a Gaussian longitudinal envelope with length $k_{i} L_{i}=32$.

and Eq. (17) yields $\sigma_{p_{x}} \simeq 0.34$. Figure 6 shows the rms transverse momentum $\sigma_{p_{x}}$ (blue points) versus normalized laser field amplitude $a_{i}$, after interaction with the ionization laser pulse calculated using the PIC code WARP in 2D with the laser polarization out of the simulation plane. Figure 6 considers an ionization laser with $\lambda_{i}=0.4 \mu \mathrm{m}$, and FWHM duration $16 \mathrm{fs}$, ionizing a krypton gas in a background plasma of density $2 \times 10^{17} \mathrm{~cm}^{-3}$. The blue dashed curve (for $a_{i}<a_{f}$ ) is Eq. (14), and the blue dotted line (for $a_{i}>a_{f}$ ) is the saturated value Eq. (17).

As Fig. 5(b) illustrates, the transverse distribution of ionized electrons will be approximately uniform, out to the threshold field amplitude for fully ionizing the gas: $a_{f}=a_{i} \exp \left(-x_{f}^{2} / w_{i}^{2}\right)$. Therefore we expect the initial rms transverse size of the distribution to be approximately

$$
\sigma_{x} \simeq x_{f} / \sqrt{3}=w_{i} \sqrt{\ln \left(a_{i} / a_{f}\right) / 3} .
$$

Figure 6 shows the rms width of the transverse distribution of ionized electrons (red circles) normalized to the laser spot size, $\sigma_{x} / w_{i}$, versus normalized laser field amplitude $a_{i}$ calculated using the PIC code WARP. The dashed red curve (for $a_{i}<a_{f}$ ) is Eq. (15), and the dotted red curve (for $a_{i}>a_{f}$ ) is Eq. (18). There is good agreement between the simple model and the PIC simulation results.

To minimize the emittance of the injected beam, it is advantageous to operate below or at the threshold for fully

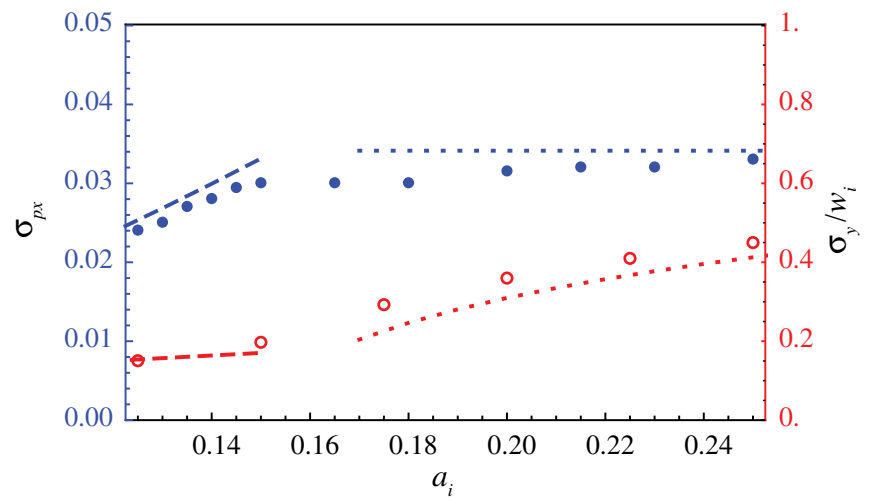

FIG. 6. The rms transverse momentum of the ionized electron distribution, after the ionization laser pulse, $\sigma_{p_{x}}$ (blue points, left vertical axis) and the rms size of the transverse spatial distribution of ionized electrons normalized to the laser spot size $\sigma_{y} / w_{i}$ (red circles, right vertical axis) obtained from PIC simulations. The ionization laser has $\lambda_{i}=0.4 \mu \mathrm{m}$, and FWHM duration $16 \mathrm{fs}$, ionizing a krypton gas, $\mathrm{Kr}^{8+} \rightarrow \mathrm{Kr}^{9+}\left(U_{I}=230 \mathrm{eV}\right)$, in a background plasma of density $2 \times 10^{17} \mathrm{~cm}^{-3}$. The blue dashed curve is Eq. (14), and the blue dotted line is the saturated value Eq. (17). The dashed red line is Eq. (15), and the red dotted line is Eq. (18).

ionizing the charge state, $a_{i} \lesssim a_{f}$, such that the transverse momentum and beam size are reduced. Further increasing the ionization laser intensity will increase the beam emittance without significantly increasing the trapped charge.

\section{E. Trapped charge}

For a sufficiently large plasma wave amplitude, an electron ionized at rest at the proper wake phase will be trapped in the plasma wave. This condition may be expressed as [18]

$$
1+\phi_{\min }-\phi\left(\psi_{i}\right) \leq \gamma_{p}^{-1}\left[1+a_{x}\left(\psi_{i}\right)^{2}\right]^{1 / 2},
$$

where $\psi_{i}=k_{p} \xi$ is the phase position in the plasma wakefield of the ionized electron, with $k_{p}^{2}=4 \pi n_{e} r_{e}$ and $n_{e}$ the electron plasma density, $\gamma_{p}$ is the phase velocity of the plasma wave, and $\phi$ is the potential of the wakefield (normalized to $m_{e} c^{2} / e$ ), with $\phi_{\min }$ the minimum amplitude of the potential. In the limits $\gamma_{p}^{2} \gg 1$ and peak accelerating wakefield $E_{m} \gg m_{e} c^{2} k_{p} / e=E_{0}, \phi_{\min } \simeq-1+\left(E_{0} / E_{m}\right)^{2}$ [1], and the wake phase region where trapping occurs is given by $\phi\left(\psi_{i}\right) \geq\left(E_{0} / E_{m}\right)^{2}$.

For an ionization laser pulse $a_{x}$ located at the proper phase $\psi_{i}$ of a plasma wave of sufficient amplitude $\phi_{\min }$, given by Eq. (19), electrons ionized at rest will be on trapped orbits, and the amount of trapped charge will be determined by the number of ionized electrons: $N_{t}=2 \pi \sigma_{x} \sigma_{y} \ell_{i} f_{i} n_{g}$, where $f_{i}=n / n_{g}$ is the ionization fraction (cf. Sec. II D) and $\ell_{i}$ is the length of the high-Z gas region. Here we assume the length of the high- $Z$ gas 
region is shorter than the ionization-laser Rayleigh range, $\ell_{i}<Z_{R i}=\pi w_{i}^{2} / \lambda_{i}$. If $\ell_{i} \gtrsim Z_{R i}$, then the total charge will be limited by the diffraction of the ionization laser.

Assuming the high- $Z$ gas is not fully ionized by a laser pulse with a Gaussian transverse profile, $\sigma_{x}$ and $\sigma_{y}$ are given by Eq. (15), and

$$
N_{t} \simeq \pi w_{i}^{2} \ell_{i} f_{i} n_{g} \Delta^{2},
$$

for $\ell_{i}<Z_{R i}$ and $\Delta^{2} \ll 1$. For example, for $\Delta=0.2, f_{i} \approx 1$, and a Gaussian laser with spot size $w_{i}=5 \mu \mathrm{m}$ propagating in a gas density of $n_{g}=2 \times 10^{17} \mathrm{~cm}^{-3}$, the trapped charge per distance propagated is $e N_{t} / \ell_{i} \simeq 57 \mathrm{pC} / \mathrm{mm}$. A higher charge can be obtained by extending the interaction distance $\ell_{i}$; however, this will result in a spread in injection times $\ell_{i} / c$ for the head and tail of the beam and, hence, a larger energy spread is produced. Equation (20) assumes all the ionized electrons are trapped, and is valid when the ionization laser pulse duration is contained in the wake phase region where electrons ionized at rest are trapped [defined by Eq. (19)] and when the trapped charge is sufficiently small such that beam loading effects may be neglected.

\section{TRANSVERSE MOMENTUM FROM PONDEROMOTIVE FORCE OF IONIZATION LASER}

Section II A described the intrinsic momentum spread in the plane of the laser owing to the quiver motion. In addition to this momentum originating from ionization off-peak of the laser electric field, an electron ionized off axis, with respect to the laser propagation discretion, will experience the transverse ponderomotive force of the ionization laser [1]: $F_{\perp} / m_{e} c^{2}=-\nabla_{\perp} \gamma_{\perp}$, where $\gamma_{\perp}^{2}=1+$ $a_{i}^{2} / 2$ and linear polarization is assumed. For a Gaussian transverse laser profile, the ponderomotive force is

$$
\frac{F_{\perp}}{m_{e} c^{2}}=\frac{a_{i}^{2}}{\gamma_{\perp}} \frac{x_{\perp}}{w_{i}^{2}} e^{-2 r^{2} / w_{i}^{2}}
$$

If the electron is ionized near the peak of the laser, $r \ll w_{i}$, the normalized momentum gained from the laser ponderomotive force is approximately

$$
u_{\perp \mathrm{PMF}} \approx\left(\sqrt{\pi} L_{i} / 2\right) \frac{a_{i}^{2}}{w_{i}^{2} \gamma_{\perp}} x_{\perp} .
$$

Equation (22) assumes $w_{i} / L_{i} \gg u_{\perp \text { PMF }}$, i.e., the ionized electron slips through the laser before being ponderomotively expelled transversely.

In the transverse plane orthogonal to the laser polarization, electrons are ionized off axis with an rms width in initial transverse position given by Eq. (15), $\sigma_{y}=$ $\sigma_{\psi} w_{i} / \sqrt{2} \simeq w_{i} \Delta / \sqrt{2}$. Hence the rms momentum gained from the ponderomotive force of the laser is

$$
\sigma_{p_{y}} \approx\left(\sqrt{\frac{\pi}{8}} \frac{L_{i}}{w_{i}}\right) \frac{a_{i}^{2}}{\gamma_{\perp}} \Delta
$$

for $\Delta^{2} \ll 1$.

The transverse momentum gained from the quiver motion in the plane of laser polarization (proportional to the longitudinal laser phase position at ionization) and the transverse position at ionization are uncorrelated $\left\langle u_{x} x\right\rangle=0$. Therefore, using Eq. (14), the rms momentum gained in the plane of laser polarization, including the ponderomotive kick from the ionization laser $\sigma_{p_{x}}=$ $\left\langle\left(u_{x}+u_{\mathrm{x} \text { PMF }}\right)^{2}\right\rangle^{1 / 2}$, is

$$
\sigma_{p_{x}}=a_{i} \Delta\left[1+\left(2 n^{*}-|m|-\frac{11}{2}\right) \Delta^{2}+\frac{\pi}{8}\left(\frac{L_{i}}{w_{i}} \frac{a_{i}}{\gamma_{\perp}}\right)^{2}\right]^{1 / 2},
$$

for $\Delta^{2} \ll 1$. The momentum gained from the transverse ponderomotive force in the plane of polarization will be an important contribution to the rms momentum spread for parameters such that $\left(L_{i} / w_{i}\right) a_{i} / \gamma_{\perp} \sim 1$.

\section{EMITTANCE}

The nature of the laser tunneling ionization mechanism sets a minimum (intrinsic) initial injected normalized beam emittance after exiting the ionization laser. In the following we assume that the ionization laser is located in the plasma wave such that ionized electrons reside on trapped and focused orbits. The momentum gained from the ponderomotive force of the ionization laser will not contribute to the initial transverse rms emittance (i.e., the thermal emittance) since the momentum gained from the ponderomotive force is correlated to the transverse position: from Eq. (22),

$$
\left\langle u_{\perp \mathrm{PMF}} x_{\perp}\right\rangle=\left(\frac{\sqrt{\pi}}{2} \frac{L_{i}}{w_{i}^{2}} \frac{a_{i}^{2}}{\gamma_{\perp}}\right)\left\langle x_{\perp}^{2}\right\rangle .
$$

In the transverse plane orthogonal to the laser polarization, the initial rms emittance after passage through the ionization laser is $\epsilon_{y}^{2}=\sigma_{p_{y}}^{2} \sigma_{y}^{2}-\left\langle y u_{\mathrm{y} \mathrm{PMF}}\right\rangle^{2}=0$.

In the plane of laser polarization, the initial rms transverse normalized emittance $\epsilon_{x}=\left[\sigma_{x}^{2} \sigma_{p_{x}}^{2}-\left\langle x\left(u_{x}+u_{\mathrm{xPMF}}\right)\right\rangle^{2}\right]^{1 / 2}$ is

$$
\epsilon_{x} \simeq a_{i} w_{i} \Delta^{2} / \sqrt{2}=\left(\frac{3 \pi r_{e}}{\sqrt{2} \alpha^{4}}\right)\left(\frac{U_{H}}{U_{I}}\right)^{3 / 2} \frac{w_{i} a_{i}^{2}}{\lambda_{i}},
$$

for $\Delta^{2} \ll 1$. Equation (26) is the thermal emittance of the beam after ionization and passage through the ionization laser pulse, in the laser polarization plane, and is a function of the ionization potential of the gas, the laser wavelength, amplitude, and spot size. Note that, for $\Delta \lesssim 1$, higher-order corrections to the emittance to order $\mathcal{O}\left(\Delta^{3}\right)$ may be included 
by retaining the corrections owing to laser envelope, the nonlinear dependence of momentum on phase position [cf. Eq. (9)], and the nonexponential dependence on the ionization rate [cf. Eq. (6)], as discussed in Sec. II.

As the trapped electrons exit the ionization laser, they begin to rotate in phase space determined by the plasma wakefield. A detailed discussion of the dynamics of electrons in a plasma wakefield is presented in Ref. [23]. In the following we will consider a linear focusing force provided by the plasma wakefield, with the betatron wave number $k_{\beta}$ related to the focusing force of the plasma wakefield: $\gamma k_{\beta}^{2} r \simeq k_{p}\left(E_{r}-B_{\theta}\right) / E_{0}$. In the bubble or blowout regime, where an ion cavity is formed by the driver, $k_{\beta}=k_{p} / \sqrt{2}$ initially (i.e., for $\gamma \simeq 1$ ).

In the plasma wakefield, the initial rms betatron amplitude of the electron beam is

$$
r_{\beta}=\left(\sigma_{\perp}^{2}+\sigma_{p_{\perp}}^{2} / k_{\beta}^{2}\right)^{1 / 2} .
$$

For a matched beam $k_{\beta} \sigma_{\perp}=\sigma_{p_{\perp}}$, and $r_{\beta}=\sqrt{2} \sigma_{\perp} \approx w_{i} \Delta$, for $\Delta^{2} \ll 1$. In general, the ionized injection beam will be mismatched to the plasma wakefield focusing forces and phase mixing will result in emittance growth [23]. As the electrons are accelerated, the betatron wave number decreases $k_{\beta} \propto \gamma^{-1 / 2}$. Provided the acceleration is adiabatic with respect to the betatron frequency, the betatron amplitude decreases $r_{\beta} \propto \gamma^{-1 / 4}$, and the normalized emittance will be preserved during acceleration: $\epsilon_{\perp}(\gamma \sim 1) \approx \epsilon_{\perp}(\gamma)$. The final transverse emittance, saturated after phase mixing via phase-space rotation, is

$$
\epsilon_{\perp}=k_{\beta} r_{\beta}^{2} / 2=\left(k_{\beta} \sigma_{\perp}^{2}+\sigma_{p_{\perp}}^{2} / k_{\beta}\right) / 2 .
$$

Consider a small amplitude ionization injection pulse $a_{i}^{2} \ll 1$ such that the ponderomotive kick may be neglected. In the limit $\Delta^{2} \ll 1$, using Eqs. (10) and (15), Eq. (28) predicts the emittance in the plane of laser polarization after saturation via phase mixing is

$$
\epsilon_{x}=\left(k_{\beta}^{2} w_{i}^{2}+2 a_{i}^{2}\right) \frac{\Delta^{2}}{4 k_{\beta}} .
$$

For $k_{\beta} \approx \sigma_{p_{x}} / \sigma_{x}$, or $a_{i} \approx\left(k_{\beta} w_{i}\right) / \sqrt{2}$, the beam is injected approximately matched to the focusing forces of the wakefield, and the intrinsic thermal emittance after ionization injection is preserved, i.e., Eq. (29) reduces to Eq. (26). Matched injection can be achieved, for example, using the two-color ionization injection scheme [22], as discussed in Sec. IVA. The emittance out of the plane of laser polarization, after saturation via phase mixing, is

$$
\epsilon_{y}=k_{\beta} w_{i}^{2} \Delta^{2} / 4 .
$$

Note that, if the high- $Z$ gas is fully ionized (e.g., as for the parameters considered in Ref. [23]) the saturated emittance is Eq. (28) with $\sigma_{p_{\perp}}$ and $\sigma_{\perp}$ given by Eqs. (17) and (18), respectively.

In the limit of sufficiently small laser amplitude such that $a_{i} \ll k_{\beta} w_{i} / 2$, the emittance is dominated by the transverse spatial distribution of the ionized electrons and the initial transverse momentum may be neglected. In this regime the transverse emittance will be approximately symmetric $\epsilon_{y} \simeq \epsilon_{x}$. Also in this limit, we expect a linear relation between the emittance and trapped charge; using Eqs. (20) and (30), the trapped charge is $N_{t}=\left(4 \pi \ell_{i} f_{i} n_{g} / k_{\beta}\right) \epsilon_{y}$.

\section{A. Case: Two-color ionization injection}

In the two-color ionization injection method [22], a longwavelength pump pulse, with large ponderomotive force and small peak electric field, excites a wake without fully ionizing a high- $Z$ gas. A short-wavelength injection pulse, with small ponderomotive force and large peak electric field, copropagating and delayed with respect to the pump laser, ionizes a fraction of the remaining bound electrons at a trapping phase of the plasma wave, generating an electron beam that is accelerated by the wake. Figure 1 shows an illustration of the two-color ionization injection geometry obtained from a 3D PIC simulation using the code WARP.

Using two-color ionization injection, a matched beam can be injected in the plane of laser polarization. For example, consider the two-color ionization case show in Fig. 1 where a plasma wake excited by a circular-polarized, $5 \mu \mathrm{m}$-wavelength laser pulse with $a_{0}=1.17,92$ fs FWHM duration, and spot size $w_{0}=36 \mu \mathrm{m}$, propagating in a plasma of density $n_{0}=2 \times 10^{17} \mathrm{~cm}^{-3} \quad\left(\lambda_{p}=75 \mu \mathrm{m}\right)$ doped with krypton gas. The pump pulse ionizes the krypton to $\mathrm{Kr}^{8+}$ without trapping electrons. A linearpolarized injection laser pulse with $a_{i}=0.135$, $w_{i}=5 \mu \mathrm{m}, \lambda_{i}=0.4 \mu \mathrm{m}$, and 16 fs FWHM duration, is delayed with respect to the pulse driving the plasma wave such that ionized electrons $\mathrm{Kr}^{8+}$ to $\mathrm{Kr}^{9+}$ are trapped in the wake. For these parameters $k_{\beta} w_{i} / \sqrt{2} \approx a_{i}$, and Eq. (26) predicts the (matched) emittance is $\epsilon_{x} \simeq 0.02 \mu \mathrm{m}$. Figure 7 shows the transverse phase space in the plane of laser polarization of the approximately matched beam obtained from a 3D PIC simulation for these parameters using the code WARP. The numerically calculated normalized transverse emittance for the simulated beam shown in Fig. 7 is $\epsilon_{x} \simeq 0.021 \mu \mathrm{m}$, in good agreement with Eq. (26). The dashed curve in Fig. 7 is the orbit in transverse phase space of an electron with initial radial position of

$$
r_{\beta}=\left[1+2 a_{i}^{2} /\left(k_{\beta} w_{i}\right)^{2}\right]^{1 / 2} w_{i} \Delta / \sqrt{2} .
$$

The matched injection shown in Fig. 7 is for the beam transverse phase space in the plane of laser polarization. Figure 8 shows the transverse phase space for the same beam in the transverse plane orthogonal to the laser 


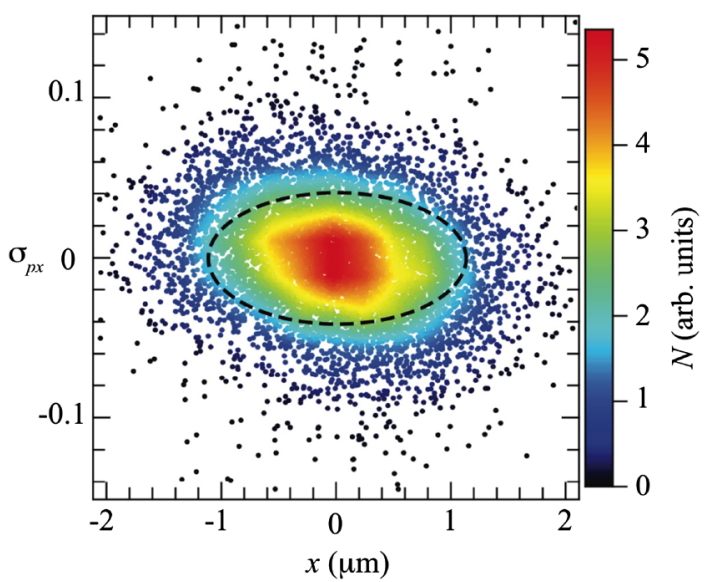

FIG. 7. Transverse phase space (in the plane of laser polarization) of ionized electrons after passage through the laser. Dashed line shows the orbit of an electron in the plasma wave with initial transverse position $r_{\beta}$. (Two-color ionization injection parameters are the same as Fig. 1.)

polarization. In the transverse plane orthogonal to the laser polarization the injected beam is mismatched to the wake focusing forces. The phase space distribution is due to the fact that the high- $\mathrm{Z}$ gas region (where ionization injection occurs) in the simulation is limited to $\ell_{i}=50 \mu \mathrm{m}$. As the laser propagates through the high- $Z$ gas region, electrons are ionized and trapped, while earlier trapped electrons rotate in phase space. The total betatron phase advance of the electrons injected first compared to the final injected electrons (after $\ell_{i}$ propagation of the laser) is $\Delta \psi_{\beta} \approx k_{\beta} \ell_{i}$. For $\Delta \psi_{\beta}<\pi$ the transverse phase space ellipse is only partially filled, as illustrated in the example shown in Fig. 8. In this case $\Delta \psi_{\beta}<\pi$, the emittance will be reduced by a factor of $\left[1-\sin ^{2}\left(\Delta \psi_{\beta}\right) /\left(\Delta \psi_{\beta}\right)^{2}\right]^{1 / 2}$ [23]. For the parameters of Fig. 8, the reduced emittance,

$$
\epsilon_{y} \simeq\left(k_{\beta} w_{i}^{2} \Delta^{2} / 4\right)\left[1-\frac{\sin ^{2}\left(k_{\beta} \ell_{i}\right)}{\left(k_{\beta} \ell_{i}\right)^{2}}\right]^{1 / 2},
$$

is $\epsilon_{y} \simeq 0.01 \mu \mathrm{m}$. The rms transverse emittance of the PIC generated beam shown in Fig. 8 is $\epsilon_{y} \simeq 0.011 \mu \mathrm{m}$, in agreement with Eq. (32) with $\ell_{i}=50 \mu \mathrm{m}$. Following the short injection region $k_{\beta} \ell_{i}<\pi$, the particle orbits will undergo betatron motion (and acceleration) in the fields of the wake. Phase mixing will occur, due to energy spread and pulse length effects, and, after a sufficiently large number of betatron periods, the projected emittance will typically saturate to Eq. (30).

Figure 9 shows the normalized emittance evolution in the laser polarization plane $\left(\epsilon_{x}\right.$, black curve) and orthogonal to the laser polarization plane $\left(\epsilon_{y}\right.$, red curve). For these parameters (same as Figs. 1, 7, and 8) the emittance of the injected beam is matched in the plane of laser polarization $\epsilon_{x} \approx$ constant. The emittance in the plane orthogonal to the laser polarization $\epsilon_{y}$ grows until the end of the high- $Z$ gas

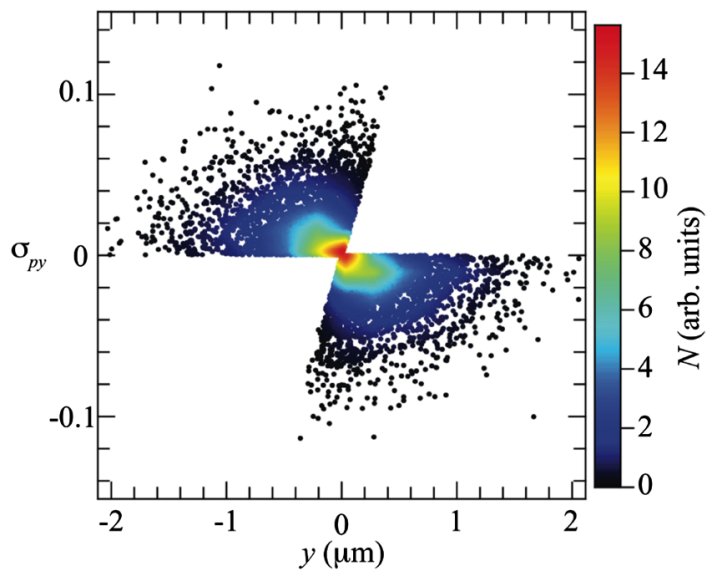

FIG. 8. Transverse phase space (out of the plane of laser polarization) of ionized electrons after ionization region $k_{\beta} L_{i} \simeq 1.9$, with transverse $\mathrm{rms}$ emittance $\epsilon_{y} \simeq 0.011 \mu \mathrm{m}$. (Two-color ionization injection parameters are the same as Fig. 1.)

region $\left(\ell_{i}=50 \mu \mathrm{m}\right)$, then reduces due to evolution of the betatron frequency as the electrons are accelerated $\left(k_{\beta} \propto \gamma^{1 / 2}\right)$ [23] (betatron phase mixing has not fully occurred over $1 \mathrm{~mm}$ of propagation). The trapped charge for the beam shown in Figs. 7 and 8 is $N_{t} \simeq 0.35 \mathrm{pC}$, in good agreement with the prediction of Eq. (20), with $\ell_{i}=50 \mu \mathrm{m}$ and $n / n_{g} \approx 0.5$.

\section{B. Case: Laser ionization injection in beam-driven plasma wakes}

Since field ionization by the beam driver can be small, this reduces the intensity required for the ionization laser. In this case, the ionization laser amplitude typically satisfies $a_{i} \ll k_{\beta} w_{i} / 2$, and the emittance is dominated by the transverse spatial distribution of the ionized electrons (with approximately symmetric transverse emittance, $\epsilon_{y} \simeq \epsilon_{x}$ ). For a plasma wakefield in the cavitated (blow-out) regime (where $k_{\beta}=k_{p} / \sqrt{2 \gamma}$ ),

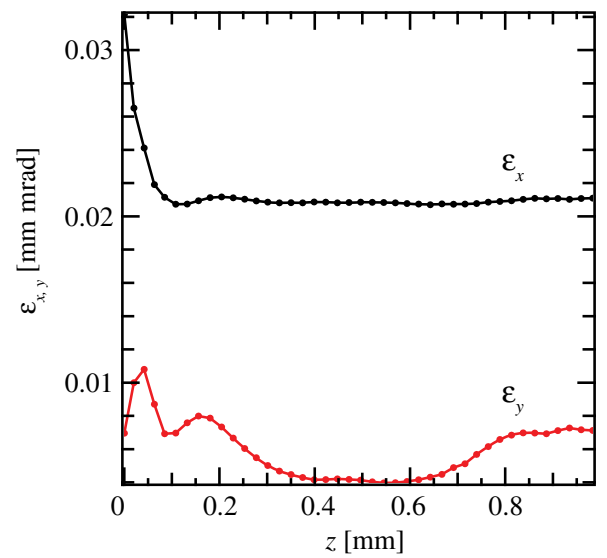

FIG. 9. The normalized emittance evolution in the laser polarization plane $\epsilon_{x}$ (black curve) and orthogonal to the laser polarization plane $\epsilon_{y}$ (red curve). (Two-color ionization injection parameters are the same as Fig. 1.) 


$$
\epsilon_{y} \simeq \epsilon_{x}=k_{p} w_{i}^{2} a_{i}\left(\frac{3 \pi r_{e}}{4 \sqrt{2} \alpha^{4} \lambda_{i}}\right)\left(\frac{U_{H}}{U_{I}}\right)^{3 / 2} .
$$

Equation (33) is typically valid for the parameter regime of the "trojan horse" scheme, as discussed in Ref. [20], where a low amplitude ionization laser pulse is delayed with respect to an electron beam driver for wakefield excitation. Using the parameters in Ref. [20], $a_{i}=0.018, w_{i}=4 \mu \mathrm{m}, U_{I}=$ $24.6 \mathrm{eV}$ (He gas), $\lambda_{i}=0.8 \mu \mathrm{m}$, and $\lambda_{p}=60 \mu \mathrm{m}$, Eq. (33) predicts $\epsilon_{x} \simeq \epsilon_{y} \simeq 0.026 \mu \mathrm{m}$. Equation (33) has the scaling $\epsilon_{y} \propto w_{i}^{2} a_{i}$, in contrast to the estimate given in Ref. [20].

The betatron wave number decreases with increasing energy as $k_{\beta} \propto \gamma^{-1 / 2}$, and, assuming adiabatic energy gain, the betatron amplitude decreases as $r_{\beta} \propto \gamma^{-1 / 4}$. The beam divergence also decreases with energy $\theta \simeq \gamma^{-3 / 4} k_{\beta} r_{\beta}$. In the limit of $a_{i} \ll k_{\beta} w_{i} / 2$ and in the cavitated regime, the beam divergence is

$$
\theta \simeq \gamma^{-3 / 4} k_{p} w_{i} \Delta / 2
$$

Using the parameters in Ref. [20], $a_{i}=0.018, w_{i}=4 \mu \mathrm{m}$, $U_{I}=24.6 \mathrm{eV}$ (He gas), $\lambda_{i}=0.8 \mu \mathrm{m}$, and $\lambda_{p}=60 \mu \mathrm{m}$, Eq. (34) predicts a divergence of $\theta \approx 1.1 \mathrm{mrad}$ at an energy of $108 \mathrm{MeV}$ (in close agreement with the simulation in Ref. [20]).

\section{SUMMARY AND CONCLUSIONS}

In this work we have derived the thermal emittance of an electron beam obtained from ionization injection in a plasma-based accelerator. The initial transverse phase space distribution created from laser tunneling ionization was calculated and compared to PIC simulations for a range of laser intensities, spot sizes, wavelengths, and gases. From the initial beam transverse phase space distribution after ionization and passage through the laser, the thermal emittance was calculated. A general expression was presented for the saturated (after phase mixing) transverse beam emittance both in and out of the laser polarization plane. The emittance is shown to be a function of the plasma density, laser wavelength, field amplitude, spot size, and ionization potential of the gas. The expressions for the injected normalized transverse emittance were compared to simulation results and found to be in good agreement.

The transverse normalized emittance after phase mixing is $\epsilon_{\perp}=k_{\beta} r_{\beta}^{2} / 2 \sim k_{\beta} w_{i}^{2} \Delta^{2} / 4$ (for a small amplitude ionization injection laser, $\left.a_{i}<k_{\beta} w_{i}\right)$. For a fixed rate of ionization, $U_{I}^{3 / 2} \lambda_{i} / a_{i} \approx$ constant and $\Delta^{2} \approx$ constant. For example, a laser, with $a_{i}=0.01$ and $\lambda_{i}=0.8 \mu \mathrm{m}$, ionizing helium to $\mathrm{He}^{+}\left(U_{I}=24.6 \mathrm{eV}\right)$, as in the example of ionization injection in a beam-driven wake (cf. Sec. IV B) and a laser, with $a_{i}=0.15$ and $\lambda_{i}=0.4 \mu \mathrm{m}$, ionizing krypton to $\mathrm{Kr}^{9+}\left(U_{I}=230 \mathrm{eV}\right)$, as in the example of ionization injection in a laser-driven wake (cf. Sec. IVA), both yield $\Delta \simeq 0.22$. Although the quiver momentum $a_{i}$ is an order of magnitude smaller for the case of helium ionization, we expect a similar beam emittance can be achieved in the plasma accelerator for a given injection laser spot size $\left(w_{i}\right)$ and plasma wakefield amplitude $\left(k_{\beta}\right)$.

To minimize the emittance of the injected beam, it is advantageous to operate at the threshold for fully ionizing the charge state, such that the transverse momentum and beam size are reduced. Further increasing the ionization laser intensity will increase the beam emittance without significantly increasing the trapped charge. In this regime the trapped charge is linearly proportional to the emittance, $\epsilon_{\perp}=k_{\beta} r_{\beta}^{2} / 2 \sim k_{\beta} w_{i}^{2} \Delta^{2} / 4 \propto N_{t}$. For fixed ionization rate (fixed $\Delta$ ) and fixed plasma focusing (fixed $k_{\beta}$ ), the emittance may be reduced by decreasing the ionization laser spot size, and further minimization of the emittance will be limited by the focusability of the laser, $w_{i}>\lambda_{i}$. The beam charge may be increased by increasing the length of the high- $Z$ gas region; however, increasing the ionization region will result in larger energy spreads and, for sufficiently high beam charge, beam loading will become important.

The general results presented in this work can be applied to a variety of ionization injection schemes and geometries in plasma-based accelerators, independent of the wakefield driver, and enable the design and optimization of plasmabased accelerators using ionization injection for the generation of ultralow emittance beams.

\section{ACKNOWLEDGMENTS}

This work was supported by the Director, Office of Science, Office of High Energy Physics, of the U.S. Department of Energy under Contracts No. DE-AC0205CH11231 and No. DE-FG02-12ER41798, and by the DOE SCIDAC program COMPASS. This research used computational resources of the National Energy Research Scientific Computing Center, which is supported by the Office of Science of the U.S. Department of Energy under Contract No. DE-AC02-05CH11231. This work was supported in part by the National Basic Research Program of China (Grant No. 2013CBA01504) and the National Natural Science Foundation of China (Grant No. 11405107).

[1] E. Esarey, C. B. Schroeder, and W. P. Leemans, Rev. Mod. Phys. 81, 1229 (2009).

[2] O. Lundh, J. Lim, C. Rechatin, L. Ammoura, A. Ben-Ismail, X. Davoine, G. Gallot, J. Goddet, E. Lefebvre, V. Malka, and J. Faure, Nat. Phys. 7, 219 (2011); A. Buck, M. Nicolai, K. Schmid, C. M. S. Sears, A. Sävert, J. M. Mikhailova, F. Krausz, M. C. Kaluza, and L. Veisz, Nat. Phys. 7, 543 (2011).

[3] W. P. Leemans, B. Nagler, A. J. Gonsalves, C. Tóth, K. Nakamura, C. G. R. Geddes, E. Esarey, C. B. Schroeder, and S. M. Hooker, Nat. Phys. 2, 696 (2006). 
[4] A. Pukhov and J. Meyer-ter-Vehn, Appl. Phys. B 74, 355 (2002).

[5] C. Benedetti, C. B. Schroeder, E. Esarey, F. Rossi, and W. P. Leemans, Phys. Plasmas 20, 103108 (2013).

[6] G. R. Plateau, C. G. R. Geddes, D. B. Thorn, M. Chen, C. Benedetti, E. Esarey, A. J. Gonsalves, N. H. Matlis, K. Nakamura, C. B. Schroeder, S. Shiraishi, T. Sokollik, J. van Tilborg, Cs. Tóth, S. Trotsenko, T. S. Kim, M. Battaglia, T. Stöhlker, and W. P. Leemans, Phys. Rev. Lett. 109, 064802 (2012); R. Weingartner, S. Raith, A. Popp, S. Chou, J. Wenz, K. Khrennikov, M. Heigoldt, A. R. Maier, N. Kajumba, M. Fuchs, B. Zeitler, F. Krausz, S. Karsch, and F. Grüner, Phys. Rev. ST Accel. Beams 15, 111302 (2012).

[7] H.-P. Schlenvoigt, K. Haupt, A. Debus, F. Budde, O. Jäckel, S. Pfotenhauer, H. Schwoerer, E. Rohwer, J. G. Gallacher, E. Brunetti, R. P. Shanks, S. M. Wiggins, and D. A. Jaroszynski, Nat. Phys. 4, 130 (2008); M. Fuchs, R. Weingartner, A. Popp, Zs. Major, S. Becker, J. Osterhoff, I. Cortrie, R. Hörlein, G. D. Tsakiris, U. Schramm, T. P. Rowlands-Rees, S. M. Hooker, D. Habs, F. Krausz, S. Karsch, and F. Grüner, Nat. Phys. 5, 826 (2009).

[8] A. R. Maier, A. Meseck, S. Reiche, C. B. Schroeder, T. Seggebrock, and F. Grüner, Phys. Rev. X 2, 031019 (2012); Z. Huang, Y. Ding, and C. B. Schroeder, Phys. Rev. Lett. 109, 204801 (2012).

[9] N. D. Powers, I. Ghebregziabher, G. Golovin, C. Liu, S. Chen, S. Banerjee, J. Zhang, and D. P. Umstadter, Nat. Photonics 8, 28 (2014).

[10] E. Esarey, R. F. Hubbard, W. P. Leemans, A. Ting, and P. Sprangle, Phys. Rev. Lett. 79, 2682 (1997); C. B. Schroeder, P. B. Lee, J. S. Wurtele, E. Esarey, and W. P. Leemans, Phys. Rev. E 59, 6037 (1999)G. Fubiani, E. Esarey, C. B. Schroeder, and W. P. Leemans, Phys. Rev. E 70, 016402 (2004); H. Kotaki, S. Masuda, M. Kando, J. K. Koga, and K. Nakajima, Phys. Plasmas 11, 3296 (2004); M. Chen, E. Esarey, C. G. R. Geddes, E. CormierMichel, C. B. Schroeder, S. S. Bulanov, C. Benedetti, L. L. Yu, S. Rykovanov, D. L. Bruhwiler, and W. P. Leemans, Phys. Rev. ST Accel. Beams 17, 051303 (2014).

[11] J. Faure, C. Rechatin, A. Norlin, A. Lifschitz, Y. Glinec, and V. Malka, Nature (London) 444, 737 (2006); C. Rechatin, J. Faure, A. Ben-Ismail, J. Lim, R. Fitour, A. Specka, H. Videau, A. Tafzi, F. Burgy, and V. Malka, Phys. Rev. Lett. 102, 164801 (2009); H. Kotaki, I. Daito, M. Kando, Y. Hayashi, K. Kawase, T. Kameshima, Y. Fukuda, T. Homma, J. Ma, L.-M. Chen, T. Z. Esirkepov, A. S. Pirozhkov, J. K. Koga, A. Faenov, T. Pikuz, H. Kiriyama, H. Okada, T. Shimomura, Y. Nakai, M. Tanoue, H. Sasao, D. Wakai, H. Matsuura, S. Kondo, S. Kanazawa, A. Sugiyama, H. Daido, and S. V. Bulanov, Phys. Rev. Lett. 103, 194803 (2009).

[12] S. Bulanov, N. Naumova, F. Pegoraro, and J. Sakai, Phys. Rev. E 58, R5257 (1998); H. Suk, N. Barov, J. B.
Rosenzweig, and E. Esarey, Phys. Rev. Lett. 86, 1011 (2001).

[13] C. G. R. Geddes, K. Nakamura, G. R. Plateau, Cs. Tóth, E. Cormier-Michel, E. Esarey, C. B. Schroeder, J. R. Cary, and W. P. Leemans, Phys. Rev. Lett. 100, 215004 (2008); K. Schmid, A. Buck, C. M. S. Sears, J. M. Mikhailova, R. Tautz, D. Herrmann, M. Geissler, F. Krausz, and L. Veisz, Phys. Rev. ST Accel. Beams 13, 091301 (2010); J. Faure, C. Rechatin, O. Lundh, L. Ammoura, and V. Malka, Phys. Plasmas 17, 083107 (2010).

[14] D. Umstadter, J. K. Kim, and E. Dodd, Phys. Rev. Lett. 76, 2073 (1996).

[15] M. Chen, Z.-M. Sheng, Y.-Y. Ma, and J. Zhang, J. Appl. Phys. 99, 056109 (2006).

[16] A. Pak, K. A. Marsh, S. F. Martins, W. Lu, W. B. Mori, and C. Joshi, Phys. Rev. Lett. 104, 025003 (2010); C. McGuffey, A. G. R. Thomas, W. Schumaker, T. Matsuoka, V. Chvykov, F. J. Dollar, G. Kalintchenko, V. Yanovsky, A. Maksimchuk, K. Krushelnick, V. Y. Bychenkov, I. V. Glazyrin, and A. V. Karpeev, Phys. Rev. Lett. 104, 025004 (2010).

[17] J. S. Liu, C. Q. Xia, W. T. Wang, H. Y. Lu, C. Wang, A. H. Deng, W. T. Li, H. Zhang, X. Y. Liang, Y. X. Leng, X. M. Lu, C. Wang, J. Z. Wang, K. Nakajima, R. X. Li, and Z. Z. Xu, Phys. Rev. Lett. 107, 035001 (2011); B. B. Pollock, C. E. Clayton, J. E. Ralph, F. Albert, A. Davidson, L. Divol, C. Filip, S. H. Glenzer, K. Herpoldt, W. Lu, K. A. Marsh, J. Meinecke, W. B. Mori, A. Pak, T. C. Rensink, J. S. Ross, J. Shaw, G. R. Tynan, C. Joshi, and D. H. Froula, Phys. Rev. Lett. 107, 045001 (2011).

[18] M. Chen, E. Esarey, C. B. Schroeder, C. G. R. Geddes, and W. P. Leemans, Phys. Plasmas 19, 033101 (2012).

[19] N. Bourgeois, J. Cowley, and S. M. Hooker, Phys. Rev. Lett. 111, 155004 (2013).

[20] B. Hidding, G. Pretzler, J. B. Rosenzweig, T. Königstein, D. Schiller, and D. L. Bruhwiler, Phys. Rev. Lett. 108, 035001 (2012).

[21] Y. Xi, B. Hidding, D. Bruhwiler, G. Pretzler, and J. B. Rosenzweig, Phys. Rev. ST Accel. Beams 16, 031303 (2013).

[22] L.-L. Yu, E. Esarey, C. B. Schroeder, J.-L. Vay, C. Benedetti, C. G. R. Geddes, M. Chen, and W. P. Leemans, Phys. Rev. Lett. 112, 125001 (2014).

[23] X. L. Xu, J. F. Hua, F. Li, C. J. Zhang, L. X. Yan, Y. C. Du, W. H. Huang, H. B. Chen, C. X. Tang, W. Lu, P. Yu, W. An, C. Joshi, and W. B. Mori, Phys. Rev. Lett. 112, 035003 (2014).

[24] J. B. Rosenzweig, B. Breizman, T. Katsouleas, and J. J. Su, Phys. Rev. A 44, R6189 (1991).

[25] V. S. Popov, Phys. Usp. 47, 855 (2004).

[26] A. Pukhov, J. Plasma Phys. 61, 425 (1999).

[27] M. Chen, E. Cormier-Michel, C. Geddes, D. L. Bruhwiler, L. L. Yu, E. Esarey, C. B. Schroeder, and W. P. Leemans, J. Comput. Phys. 236, 220 (2013).

[28] J.-L. Vay, D. P. Grote, R. H. Cohen, and A. Friedman, Comput. Sci. Discovery 5, 014019 (2012). 